\title{
Review Article \\ Repulsive Guidance Molecule-a and Central Nervous System Diseases
}

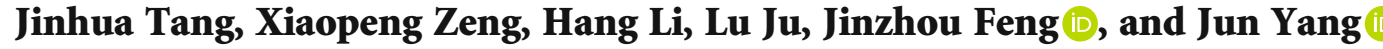 \\ Department of Neurology, The First Affiliated Hospital of Chongqing Medical University, Chongqing 400016, China \\ Correspondence should be addressed to Jinzhou Feng; fengjinzhou@hotmail.com and Jun Yang; yangweixiao222@sina.com
}

Received 5 February 2021; Accepted 27 April 2021; Published 4 May 2021

Academic Editor: Yuzhen Xu

Copyright (C) 2021 Jinhua Tang et al. This is an open access article distributed under the Creative Commons Attribution License, which permits unrestricted use, distribution, and reproduction in any medium, provided the original work is properly cited.

\begin{abstract}
Repulsive guidance molecule-a (RGMa) is a member of glycosylphosphatidylinositol- (GPI-) anchored protein family, which has axon guidance function and is widely involved in the development and pathological processes of the central nervous system (CNS). On the one hand, the binding of RGMa and its receptor Neogenin can regulate axonal guidance, differentiation of neural stem cells into neurons, and the survival of these cells; on the other hand, RGMa can inhibit functional recovery of CNS by inhibiting axonal growth. A number of studies have shown that RGMa may be involved in the pathogenesis of CNS diseases, such as multiple sclerosis, neuromyelitis optica spectrum diseases, cerebral infarction, spinal cord injury, Parkinson's disease, and epilepsy. Targeting RGMa can enhance the functional recovery of CNS, so it may become a promising target for the treatment of CNS diseases. This article will comprehensively review the research progression of RGMa in various CNS diseases up to date.
\end{abstract}

\section{Introduction}

RGMa (repulsive guidance molecule-a) is a member of glycosylphosphatidylinositol (GPI)-anchored protein family [1]. It was first found in the visual system of chicken embryo, with axon guidance function [1]. Three members of the RGM family, RGMa, RGMb, and RGMc were found in vertebrates [2]. Their 3D structures have been partially discovered [2,3]. RGMa and RGMb are expressed in the central nervous system (CNS) and other tissues (heart, lung, liver, small intestine, etc.) with a nonoverlapping form, while RGMc is only expressed in the skeletal muscle, liver, and blood [2]. The RGMa gene is located on chromosome 15q26.1 and encodes a protein of 450 amino acids [1,2]. RGMa consists of GPI-anchored C-terminal signal peptide, N-terminal signal peptide, and RGD motif (only found in RGMa and RGMc) and partial von Willebrand factor type $\mathrm{D}[2,4,5]$. RGMa exists in CNS such as neural stem cells, neuron cells, and myelin sheath in both soluble and membrane-bound forms $[4,5]$. It binds with type I transmembrane protein Neogenin and plays the biological functions of axon guidance and neuron survival through the FAK-RhoA signaling pathway $[4,5]$. In addition, as a coreceptor of bone morphogenetic proteins (BMPs), RGMa can bind to BMP2, BMP-4, and other BMP family molecules and participate in iron metabolism, bone development, and axon regeneration through the BMP-BMPR signaling pathway $[2,5]$. Currently, a number of studies have shown that RGMa is highly expressed in the injured lesions in patients with multiple sclerosis, neuromyelitis optica spectrum diseases, cerebral infarction, spinal cord injury, and Parkinson's disease [6-11]. It has also been reported that it can promote the functional recovery of the nervous system by inhibiting RGMa [6-11]. However, the expression of RGMa is low in patients with epilepsy [12]. Upregulation of RGMa can reduce epileptic seizures [12]. These data indicated that RGMa may be involved in the pathogenesis of the above diseases and may become a potential target for the treatment of CNS diseases.

\section{Role of RGMa in CNS Physiology}

2.1. Cell Proliferation and Differentiation. Both RGMa and Neogenin are highly expressed in intestinal neural stem cells during proliferation and differentiation [13]. The loss of 
(a)

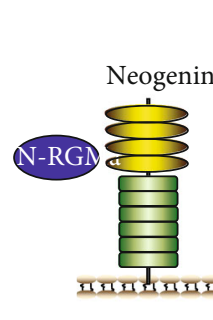

(b) (c)

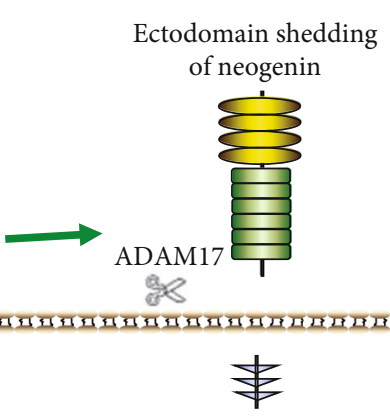

Ectodomain shedding

(d)

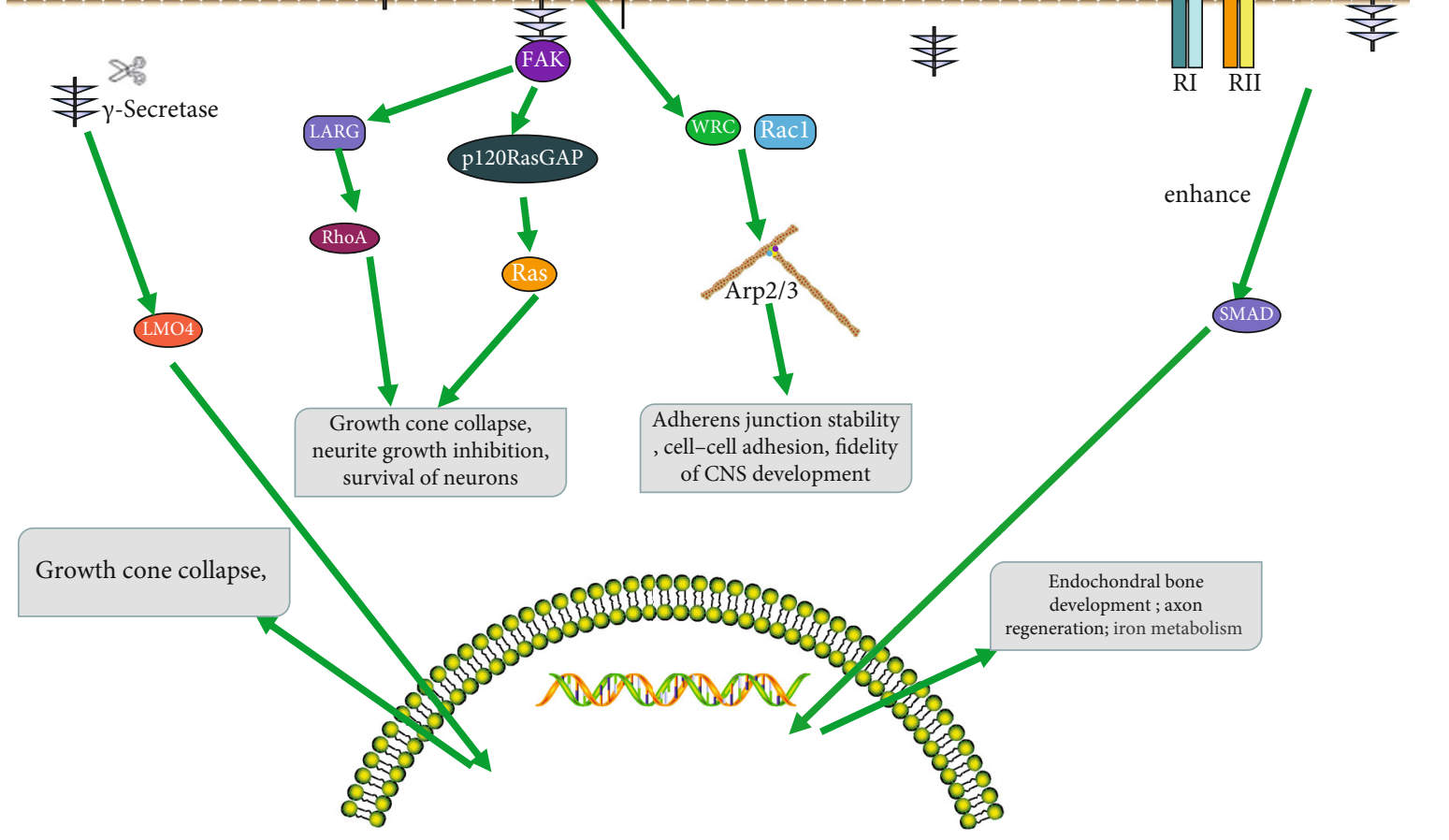

Figure 1: Mechanisms for RGMa Signal Transduction. (a) The role of N-RGM depends on the release of Neogenin intracellular domain by $\gamma$-secretase and LMO4. It has been suggested that Neogenin intracellular domain may enter the nucleus together with LMO4 and regulates gene transcription and growth cone collapse. (b) In general, C-RGM-Neogenin binding can activate RhoA through Unc5 and LARG and inactivate Ras through FAK and p120 RasGAP, thus inducing growth cone collapse and playing the role of axon guidance and regulation of neuronal survival. The binding of C-RGMa with Neogenin inhibits the interaction between Lrig2 and Neogenin. At this time, ADAM17 can cleave Neogenin and cause the extracellular domain of Neogenin to fall off, eventually leading to signal termination. Therefore, LRIG2 and ADAM17 can regulate the sensitivity of neurons to RGMa. (c) In epithelial cells, Neogenin binds to and localizes the wave regulatory complex (WRC), leading to actin nucleation via Arp2/3, which also requires Racl to activate the stability of adhesion junctions. (d) RGMa acts as a coreceptor of bone morphogenetic protein (BMP) and has been proposed as a structural bridge between BMP and Neogenin. A recently proposed model suggests that RGMa induces endocytosis of BMP receptor complexes, thereby activating classical Smad signaling. The interaction between RGM and BMP signal transduction has been involved in iron metabolism, bone development, axon regeneration, and so on.

RGMa and Neogenin resulted in the decrease of neurons, glial cells, and ganglia in the intestinal system, indicating that RGMa is involved in the proliferation and differentiation of intestinal neural stem cells [13]. RGMa had a rejection effect on differentiating progenitors via Neogenin $[13,14]$. In the midbrain of a chicken embryo, RGMa overexpression can temporarily inhibit cell proliferation [15]. In addition, RGMa promotes neuronal differentiation in the midbrain through Neogenin signal transduction [15].

2.2. Cell Adhesion and Migration. RGMa can improve the adhesion of embryonic cells in vitro, and RGMa overexpression can also induce the migration defects in early embryonic ectodermal cells, indicating that RGMa is related to the adhesion and migration of embryonic cells [16]. RGMa increases the adhesion between cells through Neogenin, recruiting cell adhesion molecules [17]. Moreover, RGMa and Neogenin jointly act on adhesion junctions (AJ) to regulate actin and maintain epithelial fidelity. In epithelial cells, Neogenin binds to and localizes the wave regulatory complex (WRC), leading to actin nucleation via Arp2/3 (Figure 1(c)) [2]. RGMa induces cell migration through Neogenin, independent of the BMP pathway [17]. Recent studies have shown that Neogenin-Netrin-1-RGMa complex regulates neuron migration [18]. The vWF and RGD domains in RGMa play a functional role in cell adhesion and cell migration [17].

2.3. Neurogenesis and Neural Tube Closure. RGMa and Neogenin can coregulate the differentiation and migration of embryonic neurogenesis, and they can also coregulate the 
development of an adult central nervous system (Figures 1(a) and $1(\mathrm{~b}))[15,17]$. In addition, RGMa can induce Neogenin protein hydrolysis and promote neural tube morphogenesis [19]. The closure defects of neural tube may occur if RGMa is exhausted [19]. Neogenin-Netrin-1-RGMa complex may regulate neurogenesis and neural tube closure through the RhoA/ROCK pathway [18]. In addition, whether the RGMa-BMP pathway plays a role in neurogenesis is a hot topic in future research [17].

\section{Role of RGMa in CNS Pathology}

3.1. Survival of Neurons. On the one hand, inhibition of RGMa in adult dentate gyrus can increase the number of new neurons; on the other hand, inhibition of Neogenin can improve neuron survival and behavioral recovery after spinal cord injury $[20,21]$. It has been proved that both RGMa and Neogenin regulate the survival of neurons $[20,21]$. Some scholars believe that RGMa binds to Neogenin on neural stem cells and regulates neuron survival by regulating caspase- 3 and Rock [15, 17]. Other scholars believe that the combination of RGMa and Neogenin affects the survival of neurons in the CNS through deathassociated protein kinase (DAPK) and LMO4. DAPK affects cell survival by activating the apoptotic pathway, while LMO4 affects the cytoskeleton and gene expression $[2,22]$.

3.2. Synapse Formation. RGMa can inhibit synapse formation by interfering with the expression of presynaptic protein synapsin-1 and postsynaptic protein PSD-95 in cortical neurons [23]. Inhibition of RGMa can increase the coexpression of the above two proteins, thus enhancing the synaptic formation after spinal cord injury [23]. RGMa, especially C-RGMa, may inhibit synaptic formation through Neogenin [24]. The latest research shows that RGMa can regulate neuronal branching through the RhoA pathway to mediate synaptic plasticity [25].

3.3. Growth Cone Collapse and Axon Growth Inhibition. RGMa can inhibit the axon growth after CNS injury, but the specific mechanism is not clear. It may inhibit the axon growth by stimulating neurons to induce RhoA and ROCK (Rho-associated coiled-coil protein kinase) expression [5]. It has been confirmed that RGMa may cause cone collapse by activating downstream Rho-GTPase activity [26]. In addition, RGMa can regulate the phosphorylation of collapse response mediator protein-2 (CRMP-2) by activating Rhokinase and glycogen synthase kinase $3 \beta$ (GSK-3 $\beta$ ) signaling pathways, thus regulating the axonal shortening [26]. Two independent c-RGMa and n-RGMa can activate different intracellular pathways to regulate neuronal survival: (1) in general, c-RGMa combines with Neogenin to activate RhoA through Unc5 and LARG (Figure 1(b)) [27-29]. C-RGMa can inhibit axon growth through the Rho-GEF (LARG)/ Rho/ROCK signaling pathway and also inactivate Ras through FAK and p120 Ras-GAP to induce growth collapse (Figure 1(b)) [27-29]. (2) When C-RGMa and Neogenin bind to inhibit the interaction between LRIG2 and Neogenin, then, C-RGMa can promote ADAM17 specific cleavage of
Neogenin, resulting in signal termination (Figure 1(b)) [27-29]. (3) N-RGMa mainly depends on $\gamma$-secretase to cleave the intracellular part of Neogenin to generate intracellular domain [27]. This domain can inhibit axonal growth by binding to LIM protein 4 (LMO4) (Figure 1(a)) [27]. Inhibition of RGMa with specific antibodies can promote axonal germination, regeneration, and motor recovery after spinal cord injury (SCI) in primates [30].

3.4. Immunoregulation. RGMa can regulate $\mathrm{T}$ cell activation and autoimmunity through dendritic cells (DCS) [31]. RGMa in dendritic cells can also bind to Neogenin on $\mathrm{CD}^{+} \mathrm{T}$ cells to activate inflammatory cells, enhance the adhesion between inflammatory cells and ICAM-1, and indirectly regulate the release and diffusion of cytokines [31]. Treatment with RGMa neutralizing antibody can cause dendritic cell tolerance and immunomodulatory function; reduce the levels of $\mathrm{MHC}$ II, CD86, CD80, and CD40; decrease the levels of IL-12, IL- $1 \beta$, and TNF- $\alpha$; and increase the secretion of IL-10, resulting in reducing $\mathrm{T}$ cell proliferation and enhancing the $\mathrm{T}$ cell differentiation into $\mathrm{Th} 2$ cells [32].

3.5. Inhibition of Angiogenesis. RGMa is a negative regulator of angiogenesis [33]. The binding of recombinant RGMa with Neogenin on endothelial cells can significantly reduce endothelial cell proliferation, migration, and formation of vascular endothelium, as well as the level of phosphorylated focal adhesion kinase (p-FAK Tyr397) [5, 33, 34]. In addition, F-actin assembled in the cytoskeleton was also significantly inhibited, thereby inhibiting cytoskeleton reorganization [5]. Removal of Neogenin or Unc5b could significantly reduce the effect of RGMa [5, 34]. RGMa can inhibit angiogenesis by down-regulating VEGF and p-FAK (Tyr397) in vitro $[34,35]$. Recombinant RGMa can also inhibit angiogenesis [36].

\section{RGMa as a Therapeutic Target in CNS Disorders}

4.1. Multiple Sclerosis (MS). Many researches have shown that RGMa plays an important role in MS. Demicheva et al. reported that the expression of RGMa was significantly increased in acute and chronic damaged plaques and normal white matter of CNS in MS patients [22]. The level of RGMa in the baseline blood was negatively correlated with the changes of Expanded Disability Status Scale (EDSS) in MS patients, indicating that the level of RGMa was closely related to neurological function $[2,6,37]$. The possible pathogeneses of RGMa in MS include the following:

(1) Abnormal signal transduction of immune cells: since IL-17-expressing $\mathrm{CD}^{+}{ }^{+} \mathrm{T}$ cells (Th17 cells) strongly expressed RGMa, so the combination of RGMa and Neogenin on immune cells can enhance the immune cell adhesion, promote their invasion to the brain, and enhance $T$ cell response [5, 37]. Neutralizing RGMa antibody can reduce the severity of experimental autoimmune encephalomyelitis (EAE) in the 
MS animal model; secondly, it can inhibit peripheral blood $\mathrm{T}$ cell proliferation, block the production of inflammatory cytokines such as IL- 2 , IFN- $\gamma$, IL-17, and IL-4, and significantly reduce the level of CNS inflammatory cytokines in MS patients $[5,37]$.

(2) Promotion of demyelinating production: in EAE, RGMa promotes the demyelination of CNS by enhancing the activation of $\mathrm{CD}^{+} \mathrm{T}$ cells $[38,39]$. Our previous work also found that the usage of RGMa neutralizing antibody can reduce the demyelination level of EAE mice, thereby inhibiting the neurological damage.

(3) Promotion of neurodegeneration: RGMa has strong inhibitory activity on axon regeneration and also plays a role in MS neurodegeneration [40]. The possible mechanism involves RGMa inducing Akt dephosphorylation in neurons by binding to Neogenin on Th17 cells [40]. Neutralizing RGMa antibody can enhance the axonal regeneration ability of inflammatory lesions, reduce axonal degeneration and clinical severity, and promote the growth of corticospinal tract and motor recovery in EAE mice $[5,7,22,40]$.

(4) Inhibition of angiogenesis: angiogenesis is another key factor involved in the pathophysiology of EAE [41]. RGMa can inhibit the formation of endothelial vessels $[5,41]$.

(5) Alter the permeability of blood-brain barrier (BBB): the damage of $\mathrm{BBB}$ is an important pathological feature of MS $[42,43]$. Studies have found that the level of RGMa in cerebrospinal fluid in patients with triamcinolone acetonide treatment (its pharmacological effect is mainly on improving the BBB permeability) is reduced, which suggested that RGMa may be involved in the pathology of MS by regulating BBB permeability in MS patients (Table 1) [44, 45].

4.2. Neuromyelitis Optica Spectrum Disorders (NMOSD). Systemic administration of anti RGMa antibody can delay the onset time, alleviate its clinical symptoms, and reduce inflammatory cell infiltration and axon damage in NMOSD rat model, indicating that inhibiting RGMa can effectively treat NMOSD [8]. The possible pathogeneses of RGMa in NMOSD include the following: (1) the loss of aquaporin-4 (AQP4) and glial fibrillary acid protein (GFAP) often occurred before the demyelination of NMOSD [46]. AntiRGMa antibody could partially restore the expression of AQP4 and GFAP in NMOSD rats, resulting in preventing astrocytopenia and relieving clinical symptoms $[8,46]$. (2) Anti-RGMa antibody can reduce the immune response of NMOSD rats, which may help to delay the attack and/or progress of NMOSD in the NMOSD rat model by reducing the number of activated microglia and reducing the infiltration of IL-17A ${ }^{+} \mathrm{T}$ cells $[8,47]$. (3) Axonal injury is an early pathological feature of NMOSD, which can cause dyskinesia [40]. The treatment of anti RGMa antibody can reduce axonal degeneration and injury [8, 40, 48]. (4) Inhibition of RGMa can promote the repair of damaged neural network and delay the secondary progression of NMOSD (Table 1) [8, 49].

4.3. Cerebral Infarction. Our previous study found that an adenovirus vector can reduce $\mathrm{BBB}$ dysfunction in rats with middle cerebral artery occlusion (MCAO)/reperfusion by inducing specific RGMa silencing [9]. The possible mechanism was that RGMa participates in $\mathrm{BBB}$ injury through the CDC-42/PAK-1 pathway (Table 1) $[9,50]$. We also found that RGMa can inhibit axonal growth by phosphorylating CRMP-2 through the Rho kinase and GSK-3 $\beta$ signaling pathways (Table 1) $[51,52]$. Both RGMa and Neogenin were expressed in neurons and vessel endothelial cells after ische$\mathrm{mia} /$ reperfusion injury in rats, and angiogenesis, coupled with functional recovery, was enhanced after RGMa RNA interference against RGMa $[34,35]$. The mechanism may lie in RGMa inhibiting angiogenesis through VEGF, Ang2, Ang1, and BDNF (Table 1) [34, 35]. In addition, the increased RGMa in patients with MCAO may be related to leptomeningeal collateral damage, which can predict the pathological state of leptomeningeal collateral by measuring the expression of RGMa mRNA in the early stage of stroke (Table 1) [53].

4.4. Spinal Cord Injury (SCI). The treatment of spinal cord injury with anti-RGMa antibody can promote the recovery of hand agility and muscle strength $[10,30]$. Possibly because the inhibition of RGMa promotes the survival and regeneration of neurons, it promotes the regeneration, repairs plasticity of corticospinal tract axons, improves motor function and gait recovery, and reduces nerve pain by reducing activated microglia (Table 1) [21].

4.5. Parkinson's Disease (PD). RGMa is upregulated in the substantia nigra of Parkinson's disease patients [11]. RGMa can induce neuropathological and behavioral changes similar to Parkinson's disease [11]. If RGMa in substantia nigra dopaminergic (DA) neurons of Parkinson's disease mouse is significantly increased, it can lead to progressive dyskinesia, including motor coordination and imbalance, which is a typical manifestation of DA reduction in striatum $[11,54-56]$. The mechanism may be the selective degeneration of DA neurons and the activation of microglia and astrocytes in substantia nigra induced by elevated RGMa (Table 1) $[11,57]$. These data suggested that RGMa dysfunction plays an important role in Parkinson's disease $[11,57]$.

4.6. Epilepsy. RGMa has been considered a potential therapeutic agent for epilepsy $[12,58]$. Some studies found that the levels of RGMa are significantly decreased in both temporal lobe epilepsy patients and experimental rats [12, 58]. Some studies also confirmed that overexpression of RGMa can inhibit epileptic seizures $[12,58]$. The possible mechanisms include the following: (1) in the organ slice model of epilepsy induced by magnesium deficiency, the overexpression of RGMa can inhibit the N-methyl-D-aspartate receptor- (NMDAR-) mediated current, thereby inhibiting the overexcitation of hippocampal neurons [58-60]. (2) Lentiviral vector-induced RGMa overexpression in the 


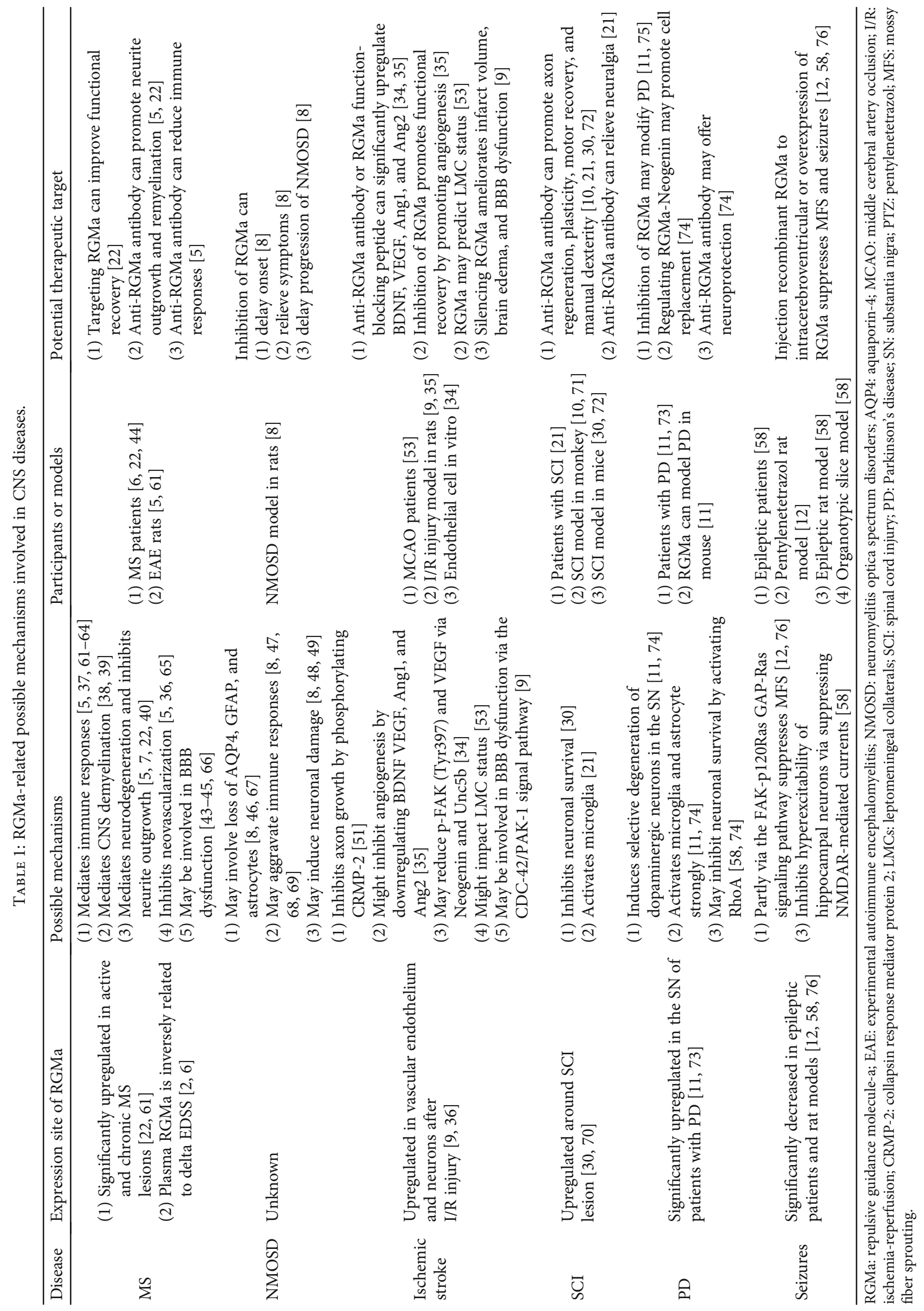


hippocampus can inhibit seizures by inhibiting mossy fiber sprouting [12]. (3) Silencing miR-20a-5p, an upstream regulator of RGMa, inhibits neuronal branching and axon growth through the RGMa-RhoA pathway, thereby preventing epilepsy (Table 1) [25].

\section{Summary}

In conclusion, as an axon guidance molecule, RGMa widely participates in the development and pathological process of CNS to regulate cell proliferation, differentiation, adhesion, migration, neurogenesis, neural tube closure, neuronal apoptosis, synapse formation, growth cone collapse, axon growth inhibition, immune response, and neovascularization through RGMa-Neogenin, RGMa-BMPs, and other signaling pathways. Recent studies have found that RGMa can participate in the pathogenesis of MS, NMOSD, cerebral infarction, spinal cord injury, PD, epilepsy, and other CNS diseases. By regulating the expression of RGMa, it can reduce neural function damage and promote the recovery of neural function, indicating that RGMa may be a promising target molecule for the treatment of CNS diseases (Table 1). As the specific pathogenesis and signaling pathway of RGMa in CNS diseases are not fully clear, the randomized controlled clinical trials need to take years to conduct. Therefore, more in-depth analysis and large sample for randomized controlled clinical trials are required to elucidate the mechanism of RGMa in the guidance of clinical treatment of CNS diseases.

\section{Conflicts of Interest}

The authors declare no conflicts of interest.

\section{Authors' Contributions}

Jinhua Tang searched the literature and wrote the manuscript, Xiaopeng Zeng drew the figure, Hang Li made the table, Lu Ju edited format, and Feng Jinzhou and Yang Jun revised the manuscript. All authors read and approved the final manuscript.

\section{Acknowledgments}

This work was supported by the National Natural Science Foundation of China (grant number 81701191).

\section{References}

[1] P. Monnier, A. Sierra, P. Macchi et al., "RGM is a repulsive guidance molecule for retinal axons," Nature, vol. 419, no. 6905, pp. 392-395, 2002.

[2] C. Siebold, T. Yamashita, P. P. Monnier, B. K. Mueller, and R. J. Pasterkamp, "RGMs: structural insights, molecular regulation, and downstream signaling," Trends in Cell Biology, vol. 27, no. 5, pp. 365-378, 2017.

[3] T. Malinauskas, T. V. Peer, B. Bishop, T. D. Mueller, and C. Siebold, "Repulsive guidance molecules lock growth differentiation factor 5 in an inhibitory complex," Proceedings of the National Academy of Sciences of the United States of America, vol. 117, no. 27, pp. 15620-15631, 2020.

[4] S. Rajagopalan, L. Deitinghoff, D. Davis et al., "Neogenin mediates the action of repulsive guidance molecule," Nature Cell Biology, vol. 6, no. 8, pp. 756-762, 2004.

[5] Y. Fujita and T. Yamashita, "The roles of RGMa-neogenin signaling in inflammation and angiogenesis," Inflammation and Regeneration, vol. 37, no. 1, p. 6, 2017.

[6] A. Malekzadeh, C. Leurs, W. van Wieringen et al., "Plasma proteome in multiple sclerosis disease progression," Annals of Clinical Translational Neurology, vol. 6, no. 9, pp. 15821594, 2019.

[7] S. Tanabe, Y. Fujita, K. Ikuma, and T. Yamashita, "Inhibiting repulsive guidance molecule-a suppresses secondary progression in mouse models of multiple sclerosis," Cell Death \& Disease, vol. 9, no. 11, p. 1061, 2018.

[8] K. Harada, Y. Fujita, T. Okuno et al., "Inhibition of RGMa alleviates symptoms in a rat model of neuromyelitis optica," Scientific Reports, vol. 8, no. 1, p. 34, 2018.

[9] M. Li, Y. Wen, R. Zhang, F. Xie, G. Zhang, and X. Qin, "Adenoviral vector-induced silencing of RGMa attenuates blood-brain barrier dysfunction in a rat model of MCAO/ reperfusion," Brain Research Bulletin, vol. 142, pp. 54-62, 2018.

[10] H. Nakagawa, T. Ninomiya, T. Yamashita, and M. Takada, "Treatment with the neutralizing antibody against repulsive guidance molecule-a promotes recovery from impaired manual dexterity in a primate model of spinal cord injury," Cerebral Cortex, vol. 29, no. 2, pp. 561-572, 2019.

[11] J. Korecka, E. Moloney, R. Eggers et al., "Repulsive guidance molecule a (RGMa) induces neuropathological and behavioral changes that closely resemble Parkinson's disease," The Journal of Neuroscience, vol. 37, no. 39, pp. 9361-9379, 2017.

[12] M. Song, F. Tian, H. Xia, and Y. Xie, "Repulsive guidance molecule a suppresses seizures and mossy fiber sprouting via the FAK-p120RasGAP-Ras signaling pathway," Molecular Medicine Reports, vol. 19, no. 4, pp. 3255-3262, 2019.

[13] M. Metzger, S. Conrad, T. Skutella, and L. Just, "RGMa inhibits neurite outgrowth of neuronal progenitors from murine enteric nervous system via the neogenin receptor in vitro," Journal of Neurochemistry, vol. 103, no. 6, pp. 2665-2678, 2007.

[14] P. H. Neckel, R. Mohr, Y. Zhang, B. Hirt, and L. Just, "Comparative microarray analysis of proliferating and differentiating murine ENS progenitor cells," Stem Cells International, vol. 2016, Article ID 9695827, 13 pages, 2016.

[15] T. J. Isaksen and T. Yamashita, "Repulsive guidance molecule a regulates adult neurogenesis via the neogenin receptor," $\mathrm{Neu}$ roscience Insights, vol. 15, article 2633105520948481, 2020.

[16] G. Lah and B. Key, "Novel roles of the chemorepellent axon guidance molecule RGMa in cell migration and adhesion," Molecular and Cellular Biology, vol. 32, no. 5, pp. 968-980, 2012.

[17] T. Isaksen, Y. Fujita, and T. Yamashita, "Repulsive guidance molecule a suppresses adult neurogenesis," Stem Cell Reports, vol. 14, no. 4, pp. 677-691, 2020.

[18] R. A. Robinson, S. C. Griffiths, L. L. van de Haar et al., "Simultaneous binding of guidance cues NET1 and RGM blocks extracellular NEO1 signaling," Cell, vol. 184, no. 8, pp. 21032120.e31, 2021.

[19] S. Brown, P. Jayachandran, M. Negesse, V. Olmo, E. Vital, and R. Brewster, "Rgma-induced Neol proteolysis promotes 
neural tube morphogenesis," The Journal of Neuroscience, vol. 39, no. 38, pp. 7465-7484, 2019.

[20] E. Matsunaga, S. Tauszig-Delamasure, P. Monnier et al., "RGM and its receptor neogenin regulate neuronal survival," Nature Cell Biology, vol. 6, no. 8, pp. 749-755, 2004.

[21] A. Mothe, N. Tassew, A. Shabanzadeh et al., "RGMa inhibition with human monoclonal antibodies promotes regeneration, plasticity and repair, and attenuates neuropathic pain after spinal cord injury," Scientific Reports, vol. 7, no. 1, article 10529, 2017.

[22] E. Demicheva, Y. Cui, P. Bardwell et al., "Targeting repulsive guidance molecule A to promote regeneration and neuroprotection in multiple sclerosis," Cell Reports, vol. 10, no. 11, pp. 1887-1898, 2015.

[23] J. Yoshida, T. Kubo, and T. Yamashita, "Inhibition of branching and spine maturation by repulsive guidance molecule in cultured cortical neurons," Biochemical and Biophysical Research Communications, vol. 372, no. 4, pp. 725-729, 2008.

[24] J. Nevoux, M. Alexandru, T. Bellocq et al., "An antibody to RGMa promotes regeneration of cochlear synapses after noise exposure," Scientific Reports, vol. 11, no. 1, p. 2937, 2021.

[25] Y. Feng, C. Duan, Z. Luo, W. Xiao, and F. Tian, "Silencing miR-20a-5p inhibits axonal growth and neuronal branching and prevents epileptogenesis through RGMa-RhoA-mediated synaptic plasticity," Journal of Cellular and Molecular Medicine, vol. 24, no. 18, pp. 10573-10588, 2020.

[26] X. Ye, Y. Qiu, Y. Gao, D. Wan, and H. Zhu, "A subtle network mediating axon guidance: intrinsic dynamic structure of growth cone, attractive and repulsive molecular cues, and the intermediate role of signaling pathways," Neural Plasticity, vol. 2019, Article ID 1719829, 26 pages, 2019.

[27] P. Banerjee, H. Harada, N. G. Tassew et al., " $\Upsilon$-secretase and LARG mediate distinct RGMa activities to control appropriate layer targeting within the optic tectum," Cell Death and Differentiation, vol. 23, no. 3, pp. 442-453, 2016.

[28] Y. Okamura, E. Kohmura, and T. Yamashita, “TACE cleaves neogenin to desensitize cortical neurons to the repulsive guidance molecule," Neuroscience Research, vol. 71, no. 1, pp. 6370, 2011.

[29] S. van Erp, D. van den Heuvel, Y. Fujita et al., "Lrig2 negatively regulates ectodomain shedding of axon guidance receptors by ADAM proteases," Developmental Cell, vol. 35, no. 5, pp. 537552, 2015.

[30] T. Nakanishi, Y. Fujita, T. Tanaka, and T. Yamashita, “Antirepulsive guidance molecule-a antibody treatment and repetitive transcranial magnetic stimulation have synergistic effects on motor recovery after spinal cord injury," Neuroscience Letters, vol. 709, article 134329, 2019.

[31] X. Xu, Y. Gao, F. Shan, and J. Feng, "A novel role for RGMa in modulation of bone marrow-derived dendritic cells maturation induced by lipopolysaccharide," International Immunopharmacology, vol. 33, pp. 99-107, 2016.

[32] X. Xu, Y. Gao, Z. Zhai, S. Zhang, F. Shan, and J. Feng, "Repulsive guidance molecule a blockade exerts the immunoregulatory function in DCs stimulated with ABP and LPS," Human Vaccines \& Immunotherapeutics, vol. 12, no. 8, pp. 21692180, 2016.

[33] W. Lu, H. Liang, Y. Li et al., "MicroRNA-210-3p targets RGMA to enhance the angiogenic functions of endothelial progenitor cells under hypoxic conditions," Frontiers in Cellular Neuroscience, vol. 13, p. 223, 2019.
[34] G. Zhang, R. Wang, K. Cheng et al., "Repulsive guidance molecule a inhibits angiogenesis by downregulating VEGF and phosphorylated focal adhesion kinase In Vitro," Frontiers in Neurology, vol. 8, p. 504, 2017.

[35] Y. Wang, R. Zhang, X. Xing et al., "Repulsive guidance molecule a suppresses angiogenesis after ischemia/reperfusion injury of middle cerebral artery occlusion in rats," Neuroscience Letters, vol. 662, pp. 318-323, 2018.

[36] K. Harada, Y. Fujita, and T. Yamashita, "Repulsive guidance molecule A suppresses angiogenesis," Biochemical and Biophysical Research Communications, vol. 469, no. 4, pp. 993999, 2016.

[37] T. Korn and A. Kallies, "T cell responses in the central nervous system," Nature Reviews. Immunology, vol. 17, no. 3, pp. 179194, 2017.

[38] T. Kubo, S. Tokita, and T. Yamashita, "Repulsive guidance molecule-a and demyelination: implications for multiple sclerosis," Journal of Neuroimmune Pharmacology, vol. 7, no. 3, pp. 524-528, 2012.

[39] R. J. M. Franklin, J. Frisén, and D. A. Lyons, "Revisiting remyelination: towards a consensus on the regeneration of CNS myelin," Seminars in Cell \& Developmental Biology, 2020.

[40] S. Tanabe and T. Yamashita, "Repulsive guidance molecule-a is involved in Th17-cell-induced neurodegeneration in autoimmune encephalomyelitis," Cell Reports, vol. 9, no. 4, pp. 1459-1470, 2014.

[41] R. Kant, S. Halder, J. Fernández, J. H. Griffin, and R. Milner, "Activated protein $\mathrm{C}$ attenuates experimental autoimmune encephalomyelitis progression by enhancing vascular integrity and suppressing microglial activation," Frontiers in Neuroscience, vol. 14, p. 333, 2020.

[42] L. Novakova, M. Axelsson, M. Khademi et al., "Cerebrospinal fluid biomarkers of inflammation and degeneration as measures of fingolimod efficacy in multiple sclerosis," Multiple Sclerosis, vol. 23, no. 1, pp. 62-71, 2017.

[43] J. Bell, J. Spencer, R. Yates, and G. DeLuca, "The cortical blood-brain barrier in multiple sclerosis: a gateway to progression?," Journal of Neurology, vol. 265, no. 4, pp. 966967, 2018.

[44] T. Müller, S. Barghorn, S. Lütge et al., "Decreased levels of repulsive guidance molecule a in association with beneficial effects of repeated intrathecal triamcinolone acetonide application in progressive multiple sclerosis patients," Journal of Neural Transmission (Vienna), vol. 122, no. 6, pp. 841-848, 2015.

[45] K. Pitarokoili, M. Sgodzai, T. Grüter et al., "Intrathecal triamcinolone acetonide exerts anti-inflammatory effects on Lewis rat experimental autoimmune neuritis and direct antioxidative effects on Schwann cells," Journal of Neuroinflammation, vol. 16, no. 1, p. 58, 2019.

[46] M. Watanabe, Y. Nakamura, Z. Michalak et al., "Serum GFAP and neurofilament light as biomarkers of disease activity and disability in NMOSD," Neurology, vol. 93, no. 13, pp. e1299e1311, 2019.

[47] E. Flanagan, P. Cabre, B. Weinshenker et al., "Epidemiology of aquaporin-4 autoimmunity and neuromyelitis optica spectrum," Annals of Neurology, vol. 79, no. 5, pp. 775-783, 2016.

[48] C. Lucchinetti, R. Mandler, D. McGavern et al., "A role for humoral mechanisms in the pathogenesis of Devic's neuromyelitis optica," Brain: A Journal of Neurology, vol. 125, no. 7, pp. 1450-1461, 2002. 
[49] H. Zhang and A. Verkman, "Eosinophil pathogenicity mechanisms and therapeutics in neuromyelitis optica," The Journal of Clinical Investigation, vol. 123, no. 5, pp. 2306-2316, 2013.

[50] L. Zhang, C. Liu, C. Huang, X. Xu, and J. Teng, "miR-155 knockdown protects against cerebral ischemia and reperfusion injury by targeting MafB," BioMed Research International, vol. 2020, Article ID 6458204, 11 pages, 2020.

[51] T. Wang, X. Wu, C. Yin, D. Klebe, J. H. Zhang, and X. Qin, "CRMP-2 is involved in axon growth inhibition induced by RGMa in vitro and in vivo," Molecular Neurobiology, vol. 47, no. 3, pp. 903-913, 2013.

[52] T. Xiong, J. Tang, J. Zhao et al., "Involvement of the Akt/GSK$3 \beta /$ CRMP-2 pathway in axonal injury after hypoxic- ischemic brain damage in neonatal rat," Neuroscience, vol. 216, pp. 123$132,2012$.

[53] Q. Wu, T. Li, B. Gong, J. Yang, and X. Qin, "Elevated repulsive guidance molecule-a mRNA in peripheral blood mononuclear cells are associated with impaired leptomeningeal collaterals in patients with middle cerebral artery occlusions," Annals of Palliative Medicine, vol. 9, no. 5, pp. 2933-2942, 2020.

[54] L. Lu, K. Xu, L. Shi et al., "Measuring Subthalamic Nucleus Volume of Parkinson's Patients and Evaluating Its Relationship with Clinical Scales at Pre- and Postdeep Brain Stimulation Treatment: A Magnetic Resonance Imaging Study," BioMed Research International, vol. 2021, Article ID 6646416, 7 pages, 2021.

[55] M. T. Gabr and S. Yahiaoui, "Multitarget therapeutics for neurodegenerative diseases," BioMed Research International, vol. 2020, Article ID 6532827, 2 pages, 2020.

[56] S. Muthuraju, R. Zakaria, M. K. M. Karuppan, and B. AlRahbi, "The role of neuroinflammation in cellular damage in neurodegenerative diseases," BioMed Research International, vol. 2020, Article ID 9231452, 2 pages, 2020.

[57] T. Müller, I. Trommer, S. Muhlack, and B. Mueller, "Levodopa increases oxidative stress and repulsive guidance molecule A levels: a pilot study in patients with Parkinson's disease," Journal of Neural Transmission, vol. 123, no. 4, pp. 401406, 2016.

[58] L. Chen, B. Gao, M. Fang et al., "Lentiviral vector-induced overexpression of RGMa in the hippocampus suppresses seizures and mossy Fiber sprouting," Molecular Neurobiology, vol. 54, no. 2, pp. 1379-1391, 2017.

[59] Y. Lu, Q. Su, M. Li et al., "Association of SCN1A, SCN2A, and UGT2B7 polymorphisms with responsiveness to valproic acid in the treatment of epilepsy," BioMed Research International, vol. 2020, Article ID 8096235, 8 pages, 2020.

[60] X. Tan, Y. Zeng, Z. Tu et al., "TRPV1 contributes to the neuroprotective effect of dexmedetomidine in pilocarpine-induced status epilepticus juvenile rats," BioMed Research International, vol. 2020, Article ID 7623635, 9 pages, 2020.

[61] R. Muramatsu, T. Kubo, M. Mori et al., "RGMa modulates T cell responses and is involved in autoimmune encephalomyelitis," Nature Medicine, vol. 17, no. 4, pp. 488-494, 2011.

[62] B. Trapp and K. Nave, "Multiple sclerosis: an immune or neurodegenerative disorder?," Annual Review of Neuroscience, vol. 31, no. 1, pp. 247-269, 2008.

[63] S. Conrad, H. Genth, F. Hofmann, I. Just, and T. Skutella, "Neogenin-RGMa signaling at the growth cone is bone morphogenetic protein- independent and involves RhoA, ROCK, and PKC," The Journal of Biological Chemistry, vol. 282, no. 22, pp. 16423-16433, 2007.
[64] R. Nohra, A. D. Beyeen, J. P. Guo et al., "RGMA_ and _IL21R_ show association with experimental inflammation and multiple sclerosis," Genes and Immunity, vol. 11, no. 4, pp. 279293, 2010.

[65] R. Muramatsu, C. Takahashi, S. Miyake, H. Fujimura, H. Mochizuki, and T. Yamashita, "Angiogenesis induced by CNS inflammation promotes neuronal remodeling through vessel-derived prostacyclin," Nature Medicine, vol. 18, no. 11, pp. 1658-1664, 2012.

[66] T. Müller, "Role of intraspinal steroid application in patients with multiple sclerosis," Expert Review of Neurotherapeutics, vol. 9, no. 9, pp. 1279-1287, 2009.

[67] T. Misu, K. Fujihara, A. Kakita et al., "Loss of aquaporin 4 in lesions of neuromyelitis optica: distinction from multiple sclerosis," Brain: A Journal of Neurology, vol. 130, no. 5, pp. 12241234, 2007.

[68] N. Asavapanumas and A. Verkman, "Neuromyelitis optica pathology in rats following intraperitoneal injection of NMO-IgG and intracerebral needle injury," Acta Neuropathologica Communications, vol. 2, no. 1, p. 48, 2014.

[69] B. Popescu, V. Lennon, J. Parisi et al., "Neuromyelitis optica unique area postrema lesions: nausea, vomiting, and pathogenic implications," Neurology, vol. 76, no. 14, pp. 12291237, 2011.

[70] J. Schwab, S. Conrad, P. Monnier, S. Julien, B. K. Mueller, and H. J. Schluesener, "Spinal cord injury-induced lesional expression of the repulsive guidance molecule (RGM)," The European Journal of Neuroscience, vol. 21, no. 6, pp. 1569-1576, 2005.

[71] H. Nakagawa and M. Takada, "Promoting functional recovery by inhibition of repulsive guidance molecule-a after spinal cord injury," Neural Regeneration Research, vol. 13, no. 6, pp. 981-982, 2018.

[72] A. Kyoto, K. Hata, and T. Yamashita, "Synapse formation of the cortico-spinal axons is enhanced by RGMa inhibition after spinal cord injury," Brain Research, vol. 1186, pp. 74-86, 2007.

[73] K. Bossers, G. Meerhoff, R. Balesar et al., "Analysis of gene expression in Parkinson's disease: possible involvement of neurotrophic support and axon guidance in dopaminergic cell death," Brain Pathology, vol. 19, no. 1, pp. 91-107, 2009.

[74] A. J. Santiago-Lopez, "A repulsive environment induces neurodegeneration of midbrain dopaminergic neurons," The Journal of Neuroscience, vol. 38, no. 6, pp. 1323-1325, 2018.

[75] E. Y. Van Battum, S. Brignani, and R. J. Pasterkamp, “Axon guidance proteins in neurological disorders," Lancet Neurology, vol. 14, no. 5, pp. 532-546, 2015.

[76] M. Y. Song, F. F. Tian, Y. Z. Wang, X. Huang, J. L. Guo, and D. X. Ding, "Potential roles of the RGMa-FAK-Ras pathway in hippocampal mossy fiber sprouting in the pentylenetetrazole kindling model," Molecular Medicine Reports, vol. 11, no. 3, pp. 1738-1744, 2015. 